\title{
Quantum Field Theory of Geometry
}

\author{
Abhay Ashtekar` and Jerzy Lewanowski ${ }^{\dagger}$ \\ ${ }^{\star}$ Center for Gravitational Physics and Geometry, \\ Physics Department, Penn State, University Park, PA 16802-6300, U.S.A. \\ $\dagger$ Institute of Theoretical Physics \\ University of Warsaw, ul Hoza 69, 00-681 Warsaw, Poland \\ ${ }^{\dagger}$ Max Planck Institut für Gravitationphysik \\ Schlaatzweg 1, 14473 Potsdam, Germany
}

\section{Introduction}

Several speakers at this conference have emphasized the conceptual difficulties of quantum gravity (see particularly [1, 2, 3]). As they pointed out, when we bring in gravity, some of the basic premises of quantum field theory have to undergo radical changes: we must learn to do physics in absence of a background space-time geometry. This immediately leads to a host of technical difficulties as well. For, the familiar mathematical methods of quantum field theory are deeply rooted in the availability of a fixed space-time metric, which, furthermore is generally taken to be flat. The purpose of this contribution is to illustrate how these conceptual and technical difficulties can be overcome.

For concreteness, we will use a specific non-perturbative approach and, furthermore, limit ourselves to just one set of issues: Exploration of the nature of quantum geometry. Nonetheless, the final results have a certain degree of robustness and the constructions involved provide concrete examples of ways in which one can analyze genuine field theories, with an infinite number of degrees of freedom, in absence of a background metric. As we will see, the underlying diffeomorphism invariance is both a curse and a blessing. On the one hand, since there is so little background structure, concrete calculations are harder and one is forced to invent new regularization methods. On the other hand, when one does succeed, the final form of results is often remarkably simple since the requirement of diffeomorphism invariance tends to restrict the answers severely. The final results are often unexpected and qualitatively different from the familiar ones from standard quantum field theories.

Let us begin with a brief discussion of the issue on which we wish to focus. In his celebrated inaugural address, Riemann suggested [4 that geometry of space may be more than just a fiducial, mathematical entity serving as a passive stage for physical phenomena, and may in fact have direct physical meaning in its own

\footnotetext{
${ }^{1}$ Invited talk at the March '96 Boston Conference on Historical Examination and Philosophical Reflections On the Foundations of Quantum Field Theory (presented by AA).
} 
right. As we know, general relativity provided a brilliant confirmation of this vision: Einstein's equations put geometry on the same footing as matter. Now, the physics of this century has shown us that matter has constituents and the 3-dimensional objects we perceive as solids in fact have a discrete underlying structure. The continuum description of matter is an approximation which succeeds brilliantly in the macroscopic regime but fails hopelessly at the atomic scale. It is therefore natural to ask if the same is true of geometry. Does geometry also have constituents at the Planck scale? What are its atoms? Its elementary excitations? Is the spacetime continuum only a 'coarse-grained' approximation? If so, what is the nature of the underlying quantum geometry?

To probe such issues, it is natural to look for hints in the procedures that have been successful in describing matter. Let us begin by asking what we mean by quantization of physical quantities. Take a simple example -the hydrogen atom. In this case, the answer is clear: while the basic observables -energy and angular momentum- take on a continuous range of values classically, in quantum mechanics their spectra are discrete. So, we can ask if the same is true of geometry. Classical geometrical observables such as areas of surfaces and volumes of regions can take on continuous values on the phase space of general relativity. Are the spectra of corresponding quantum operators discrete? If so, we would say that geometry is quantized.

Thus, it is rather easy to pose the basic questions in a precise fashion. Indeed, they could have been formulated soon after the advent of quantum mechanics. Answering them, on the other hand, has proved to be surprisingly difficult. The main reason, we believe, is the inadequacy of the standard techniques. More precisely, in the traditional approaches to quantum field theory, one begins with a continuum, background geometry. To probe the nature of quantum geometry, on the other hand, we should not begin by assuming the validity of this model. We must let quantum gravity decide whether this picture is adequate at the Planck scale; the theory itself should lead us to the correct microscopic model of geometry.

With this general philosophy, in this talk we will use a non-perturbative, canonical approach to quantum gravity to probe the nature of quantum geometry. In this approach, one uses $S U(2)$ connections on a 3-manifold as configuration variables; 3-metrics are constructed from 'electric fields' which serve as the conjugate momenta. These are all dynamical variables; to begin with, we are given only a 3-manifold without any fields. Over the past three years, this approach has been put on a firm mathematical footing through the development of a new functional calculus on the space of gauge equivalent connections [4-12]. This calculus does not use any background fields (such as a metric) and is therefore well-suited for a fully non-perturbative exploration of the nature of quantum geometry.

In section 2, we will introduce the basic tools from this functional calculus and outline our general strategy. This material is then used in section 3 to discuss the main results. In particular, operators corresponding to areas of 2 -surfaces and vol- 
umes of 3-dimensional regions are regulated in a fashion that respects the underlying diffeomorphism invariance. They turn out to be self-adjoint on the underlying (kinematical) Hilbert space of states. A striking property is that their spectra are purely discrete. This indicates that the underlying quantum geometry is far from what the continuum picture might suggest. Indeed, the fundamental excitations of quantum geometry are 1-dimensional, rather like polymers, and the 3-dimensional continuum geometry emerges only on coarse graining [13, 32]. In the case of the area operators, the spectrum is explicitly known. This detailed result should have implications on the statistical mechanical origin of the black hole entropy [15, 16] and the issue is being investigated. Section 4 discusses a few ramifications of the main results.

Our framework belongs to what Carlo Rovelli referred to in his talk as 'general quantum field theory'. Thus, our constructions do not directly fall in the category of axiomatic or constructive quantum field theory and, by and large, our calculations do not use the standard methods of perturbative quantum field theory. Nonetheless, we do discuss the quantum theory of a system with an infinite number of degrees of freedom (which, moreover, is diffeomorphism covariant) and face the issues of regularization squarely. For this, we begin 'ab-initio', construct the Hilbert space of states, introduce on it well-defined operators which represent (regulated) geometric observables and examine their properties.

Details of the results discussed here can be found in [17, 18, 19]. At a conceptual level, there is a close similarity between the basic ideas used here and those used in discussions based on the 'loop representation' [13, 20, 21, 22]. (For a comparison, see [17, 19]). Indeed, the development of the functional calculus which underlies this analysis was itself motivated, in a large measure, by the pioneering work on loop representation by Rovelli and Smolin [23]. Finally, we emphasize that this is not a comprehensive survey of non-perturbative quantum gravity; our main purpose, as mentioned already, is to illustrate how one can do quantum field theory in absence of a space-time background and to point out that results can be unexpected. Indeed, even the use of general relativity as the point of departure is only for concreteness; the main results do not depend on the details of Einstein's equations."f

\section{Tools}

This section is divided in to four parts. The first summarizes the formulation of general relativity based on connections; the second introduces the quantum configu-

\footnotetext{
${ }^{2}$ Nonetheless, since there were several remarks in this conference on the viability of quantum general relativity, it is appropriate to make a small digression to clarify the situation. It is well-known that general relativity is perturbatively non-renormalizable. However, there do exist quantum field theories which share this feature with general relativity but are exactly soluble. A striking example is $(\mathrm{GN})_{3}$, the Gross-Neveau model in 3 dimensions. Furthermore, in the case of general relativity, there are physical reasons which make perturbative methods especially unsuitable. Whether quantum general relativity can exist non-perturbatively is, however, an open question. For further details and current status, see e.g. [24.)
} 
ration space; the third presents an intuitive picture of the non-perturbative quantum states and the fourth outlines our strategy to probe quantum geometry.

\subsection{From metrics to connections}

The non-perturbative approach we wish to use here has its roots in canonical quantization. The canonical formulation of general relativity was developed in the late fifties and early sixties in a series of papers by Bergmann, Dirac and Arnowitt, Deser and Misner. In this formulation, general relativity arises as a dynamical theory of 3-metrics. The framework was therefore named geometrodynamics by Wheeler and used as a basis for canonical quantization both by him and his associates and by Bergmann and his collaborators. The framework of geometrodynamics has the advantage that classical relativists have a great deal of geometrical intuition and physical insight into the nature of the basic variables -3-metrics $g_{a b}$ and extrinsic curvatures $K_{a b}$. For these reasons, the framework has played a dominant role, e.g., in numerical relativity. However, it also has two important drawbacks. First, it sets the mathematical treatment of general relativity quite far from that of theories of other interactions where the basic dynamical variables are connections rather than metrics. Second, the equations of the theory are rather complicated in terms of metrics and extrinsic curvatures; being non-polynomial, they are difficult to carry over to quantum theory with a reasonable degree of mathematical precision.

For example, consider the standard Wheeler-DeWitt equation:

$$
\left[\frac{G \hbar}{\sqrt{g}}\left(g^{a b} g^{c d}-\frac{1}{2} g^{a c} g^{b d}\right) \frac{\delta}{\delta g_{a c}} \frac{\delta}{\delta g_{b d}}-\frac{\sqrt{g}}{G \hbar} R(g)\right] \circ \Psi(g)=0
$$

where $g$ is the determinant of the 3 -metric $g_{a b}$ and $R$ its scalar curvature. As is often emphasized, since the kinetic term involves products of functional derivatives evaluated at the same point, it is ill-defined. However, there are also other, deeper problems. These arise because, in field theory, the quantum configuration space -the domain space of wave functions $\Psi$ - is larger than the classical configuration space. While we can restrict ourselves to suitably smooth fields in the classical theory, in quantum field theory, we are forced to allow distributional field configurations. Indeed, even in the free field theories in Minkowski space, the Gaussian measure that provides the inner product is concentrated on genuine distributions. This is

the reason why in quantum theory fields arise as operator-valued distributions. One would expect that the situation would be at least as bad in quantum gravity. If so, even the products of the 3-metrics that appear in front of the momenta as well as the scalar curvature in the potential term would fail to be meaningful. Thus, the left hand side of the Wheeler-DeWitt equation is seriously ill-defined and must be regularized appropriately.

However, as we just said, the problem of distributional configurations arises already in the free field theory in Minkowski space-time. There, we do know how to regularize physically interesting operators. So, why can we not just apply those 
techniques in the present context? The problem is that those techniques are tied to the presence of a background Minkowski metric. The covariance of the Gaussian measure, for example, is constructed from the Laplacian operator on a space-like plane defined by the induced metric and normal ordering and point-splitting regularizations also make use of the background geometry. In the present case, we do not have background fields at our disposal. We therefore need to find another avenue. What is needed is a suitable functional calculus -integral and differentialthat respects the diffeomorphism invariance of the theory.

What space are we to develop this functional calculus on? Recall first that, in the canonical approach to diffeomorphism invariant theories such as general relativity or supergravity, the key mathematical problem is that of formulating and solving the quantum constraints. (In Minkowskian quantum field theories, the analogous problem is that of defining the regularized quantum Hamiltonian operator.) It is therefore natural to work with variables which, in the classical theory, simplify the form of the constraints. It turns out that, from this perspective, connections are better suited than metrics[25].

We will conclude by providing explicit expressions of these connections. Recall first that in geometrodynamics we can choose as our canonical pair, the fields $\left(E_{i}^{a}, K_{a}^{i}\right)$ where $E_{i}^{a}$ is a triad (with density weight one) and $K_{a}^{i}$, the extrinsic curvature. Here $a$ refers to the tangent space of the 3-manifold and $i$ is the internal $S O(3)$-or, $S U(2)$, if we wish to consider spinorial matter-index. The triad is the square-root of the metric in the sense that $E_{i}^{a} E^{b i}=: g g^{a b}$, where $g$ is the determinant of the covariant 3-metric $g_{a b}$, and $K_{a}^{i}$ is related to the extrinsic curvature $K_{a b}$ via: $K_{a}^{i}=(1 / \sqrt{g}) K_{a b} E^{b i}$. Let us make a change of variables:

$$
\left(E_{i}^{a}, K_{a}^{i}\right) \mapsto\left(A_{a}^{i}:=\Gamma_{a}^{i}-K_{a}^{i}, E_{i}^{a}\right),
$$

where $\Gamma_{a}^{i}$ is the spin connection determined by the triad. It is not difficult to check that this is a canonical transformation on the real phase space [25, 26]. It will be convenient to regard $A_{a}^{i}$ as the configuration variable and $E_{i}^{a}$ as the conjugate momentum so that the phase space has the same structure as in the $S U(2)$ YangMills theory. The basic Poisson bracket relations are:

$$
\left\{A_{a}^{i}(x), E_{j}^{b}(y)\right\}=G \delta_{a}^{b} \delta_{j}^{i} \delta^{3}(x, y),
$$

where the gravitational constant, $G$, features in the Poisson bracket relations because $E_{i}^{a}(x)$ now has the physical dimensions of a triad rather than that of Yang-Mills electric field. In terms of these variables, general relativity has the same kinematics as Yang-Mills theory. Indeed, one of the constraints of general relativity is precisely the Gauss constraint of Yang-Mills theory. Thus, the phase spaces of the two theories are the same and the constraint surface of general relativity is embedded in that of Yang-Mills theory. Furthermore, in terms of these variables, the remaining constraints of general relativity simplify considerably. Indeed, there is a precise sense in which they are the simplest non-trivial equations one can write down in terms of 
$A_{a}^{i}$ and $E_{i}^{a}$ without reference to any background field [29]. Finally, (in the spatially compact context) the Hamiltonian of general relativity is just a linear combination of constraints.

To summarize, one can regard the space $\mathcal{A} / \mathcal{G}$ of $S U(2)$ connections modulo gauge transformations on a ('spatial') 3-manifold $\Sigma$ as the classical configuration space of general relativity.

\subsection{Quantum configuration space}

As we already indicated, in Minkowskian quantum field theories, the quantum configuration space includes distributional fields which are absent in the classical theory and physically interesting measures are typically concentrated on these 'genuinely quantum' configurations. The overall situation is the same in general relativity.

Thus, the quantum configuration space $\overline{\mathcal{A} / \mathcal{G}}$ is a certain completion of $\mathcal{A} / \mathcal{G}$ [5, 6 ]. $\overline{\mathcal{A} / \mathcal{G}}$ inherits the quotient structure of $\mathcal{A} / \mathcal{G}$, i.e., $\overline{\mathcal{A} / \mathcal{G}}$ is the quotient of the space $\overline{\mathcal{A}}$ of generalized connections by the space $\overline{\mathcal{G}}$ of generalized gauge transformations. To see the nature of the generalization involved, recall first that each smooth connection defines a holonomy along paths in $\Sigma: h_{p}(A):=\mathcal{P} \exp -\int_{p} A$. Generalized connections capture this notion. That is, each $\bar{A}$ in $\overline{\mathcal{A}}$ can be defined [0, 9] as a map which assigns to each oriented path $p$ in $\Sigma$ an element $\bar{A}(p)$ of $S U(2)$ such that: i) $\bar{A}\left(p^{-1}\right)=(\bar{A}(p))^{-1}$; and, ii) $\bar{A}\left(p_{2} \circ p_{1}\right)=\bar{A}\left(p_{2}\right) \cdot \bar{A}\left(p_{1}\right)$, where $p^{-1}$ is obtained from $p$ by simply reversing the orientation, $p_{2} \circ p_{1}$ denotes the composition of the two paths (obtained by connecting the end of $p_{1}$ with the beginning of $p_{2}$ ) and $\bar{A}\left(p_{2}\right) \cdot \bar{A}\left(p_{1}\right)$ is the composition in $S U(2)$. A generalized gauge transformation is a map $g$ which assigns to each point $v$ of $\Sigma$ an $S U(2)$ element $g(x)$ (in an arbitrary, possibly discontinuous fashion). It acts on $\bar{A}$ in the expected manner, at the end points of paths: $\bar{A}(p) \rightarrow g\left(v_{+}\right)^{-1} \cdot \bar{A}(p) \cdot g\left(v_{-}\right)$, where $v_{-}$and $v_{+}$are respectively the beginning and the end point of $p$. If $\bar{A}$ happens to be a smooth connections, say $A$, we have $\bar{A}(p)=h_{p}(A)$. However, in general, $\bar{A}(p)$ can not be expressed as a path ordered exponential of a smooth 1-form with values in the Lie algebra of $S U(2)$ [6]. Similarly, in general, a generalized gauge transformation can not be represented by a smooth group valued function on $\Sigma$.

At first sight the spaces $\overline{\mathcal{A}}, \overline{\mathcal{G}}$ and $\overline{\mathcal{A} / \mathcal{G}}$ seem too large to be mathematically controllable. However, they admit three characterizations which enables one to introduce differential and integral calculus on them [5, 6, 8]. We will conclude this sub-section by summarizing the characterization -as suitable limits of the corresponding spaces in lattice gauge theory- which will be most useful for the main body of this paper.

Fix a graph $\gamma$ in the 3 -manifold $\Sigma$. In the physics terminology, one can think of a graph as a 'floating lattice', i.e., a lattice whose edges are not required to be

\footnotetext{
${ }^{3}$ For technical reasons, we will assume that all paths are analytic. An extension of the framework to allow for smooth paths is being carried out [31. The general expectation is that the main results will admit natural generalizations to the smooth category.
} 
rectangular. (Indeed, they may even be non-trivially knotted!) Using the standard ideas from lattice gauge theory, we can construct the configuration space associated with the graph $\gamma$. Thus, we have the space $\mathcal{A}_{\gamma}$, each element $A_{\gamma}$ of which assigns to every edge in $\gamma$ an element of $S U(2)$ and the space $\mathcal{G}_{\gamma}$ each element $g_{\gamma}$ of which assigns to each vertex in $\gamma$ an element of $S U(2)$. (Thus, if $N$ is the number of edges in $\gamma$ and $V$ the number of vertices, $\mathcal{A}_{\gamma}$ is isomorphic with $[S U(2)]^{N}$ and $\mathcal{G}_{\gamma}$ with $\left.[S U(2)]^{V}\right) . \mathcal{G}_{\gamma}$ has the obvious action on $\mathcal{A}_{\gamma}: A_{\gamma}(e) \rightarrow g\left(v_{+}\right)^{-1} \cdot A_{\gamma}(e) \cdot g\left(v_{-}\right)$. The (gauge invariant) configuration space associated with the floating lattice $\gamma$ is just $\mathcal{A}_{\gamma} / \mathcal{G}_{\gamma}$. The spaces $\overline{\mathcal{A}}, \overline{\mathcal{G}}$ and $\overline{\mathcal{A} / \mathcal{G}}$ can be obtained as well-defined (projective) limits of the spaces $\mathcal{A}_{\gamma}, \mathcal{G}_{\gamma}$ and $\mathcal{A}_{\gamma} / \mathcal{G}_{\gamma}[8$, 6]. Note however that this limit is not the usual 'continuum limit' of a lattice gauge theory in which one lets the edge length go to zero. Here, we are already in the continuum and have available to us all possible floating lattices from the beginning. We are just expressing the quantum configuration space of the continuum theory as a suitable limit of the configuration spaces of theories associated with all these lattices.

To summarize, the quantum configuration space $\overline{\mathcal{A} / \mathcal{G}}$ is a specific extension of the classical configuration space $\mathcal{A} / \mathcal{G}$. Quantum states can be expressed as complexvalued, square-integrable functions on $\overline{\mathcal{A} / \mathcal{G}}$, or, equivalently, as $\overline{\mathcal{G}}$-invariant squareintegrable functions on $\overline{\mathcal{A}}$. As in Minkowskian field theories, while $\mathcal{A} / \mathcal{G}$ is dense in $\overline{\mathcal{A} / \mathcal{G}}$ topologically, measure theoretically it is generally sparse; typically, $\mathcal{A} / \mathcal{G}$ is contained in a subset set of zero measure of $\overline{\mathcal{A} / \mathcal{G}}[\mathbb{B}]$. Consequently, what matters is the value of wave functions on 'genuinely' generalized connections. In contrast with the usual Minkowskian situation, however, $\overline{\mathcal{A}}, \overline{\mathcal{G}}$ and $\overline{\mathcal{A} / \mathcal{G}}$ are all compact spaces in their natural (Gel'fand) topologies [4-8]. This fact simplifies a number of technical issues.

\section{$2.3 \quad$ Hilbert space}

Since $\overline{\mathcal{A} / \mathcal{G}}$ is compact, it admits regular (Borel, normalized) measures and for every such measure we can construct a Hilbert space of square-integrable functions. Thus, to construct the Hilbert space of quantum states, we need to select a specific measure on $\overline{\mathcal{A} / \mathcal{G}}$.

It turns out that $\overline{\mathcal{A}}$ admits a measure $\mu^{o}$ that is preferred by both mathematical and physical considerations [6, 7]. Mathematically, the measure $\mu^{o}$ is natural because its definition does not involve introduction of any additional structure: it is induced on $\overline{\mathcal{A}}$ by the Haar measure on $S U(2)$. More precisely, since $\mathcal{A}_{\gamma}$ is isomorphic to $[S U(2)]^{N}$, the Haar measure on $S U(2)$ induces on it a measure $\mu_{\gamma}^{o}$ in the obvious fashion. As we vary $\gamma$, we obtain a family of measures which turn out to be compatible in an appropriate sense and therefore induce a measure $\mu^{o}$ on $\overline{\mathcal{A}}$. This measure has the following attractive properties [6]: i) it is faithful; i.e., for any continuous, non-negative function $f$ on $\overline{\mathcal{A}}, \int d \mu^{o} f \geq 0$, equality holding if and only if $f$ is identically zero; and, ii) it is invariant under the (induced) action of Diff $[\Sigma]$, the diffeomorphism group of $\Sigma$. Finally, $\mu^{o}$ induces a natural measure $\tilde{\mu}^{o}$ on $\overline{\mathcal{A} / \mathcal{G}}$ : 
$\tilde{\mu}^{o}$ is simply the push-forward of $\mu^{o}$ under the projection map that sends $\overline{\mathcal{A}}$ to $\overline{\mathcal{A} / \mathcal{G}}$. Physically, the measure $\tilde{\mu}^{o}$ is selected by the so-called 'reality conditions'. More precisely, the classical phase space admits an (over)complete set of naturally defined configuration and momentum variables which are real, and the requirement that the corresponding operators on the quantum Hilbert space be self-adjoint selects for us the measure $\tilde{\mu}^{o}$ 11.

Thus, it is natural to use $\tilde{\mathcal{H}}^{o}:=L^{2}\left(\overline{\mathcal{A} / \mathcal{G}}, d \tilde{\mu}^{o}\right)$ as our Hilbert space. Elements of $\tilde{\mathcal{H}}^{o}$ are the kinematic states; we are yet to impose quantum constraints. Thus, $\tilde{\mathcal{H}}^{o}$ is the classical analog of the full phase-space of quantum gravity (prior to the introduction of the constraint sub-manifold). Note that these quantum states can be regarded also as gauge invariant functions on $\overline{\mathcal{A}}$. In fact, since the spaces under consideration are compact and measures normalized, we can regard $\tilde{\mathcal{H}}^{o}$ as the gauge invariant sub-space of the Hilbert space $\mathcal{H}^{o}:=L^{2}\left(\overline{\mathcal{A}}, d \mu^{o}\right)$ of square-integrable functions on $\overline{\mathcal{A}}[\mathbf{7}$, [8]. In what follows, we we will often do so.

What do 'typical' quantum states look like? To provide an intuitive picture, we can proceed as follows. Fix a graph $\gamma$ with $N$ edges and consider functions $\Psi_{\gamma}$ of generalized connections of the form $\Psi_{\gamma}(\bar{A})=\psi\left(\bar{A}\left(e_{1}\right), \ldots, \bar{A}\left(e_{N}\right)\right)$ for some smooth function $\psi$ on $[S U(2)]^{N}$, where $e_{1}, \ldots, e_{N}$ are the edges of the graph $\gamma$. Thus, the functions $\Psi_{\gamma}$ know about what the generalized connections do only to those paths which constitute the edges of the graph $\gamma$; they are precisely the quantum states of the gauge theory associated with the 'floating lattice' $\gamma$. This space of states, although infinite dimensional, is quite 'small' in the sense that it corresponds to the Hilbert space associated with a system with only a finite number of degrees of freedom. However, if we vary $\gamma$ through all possible graphs, the collection of all states that results is very large. Indeed, one can show that it is dense in the Hilbert space $\mathcal{H}^{\circ}$. (If we restrict ourselves to $\Psi_{\gamma}$ which are gauge invariant, we obtain a dense sub-space in $\tilde{\mathcal{H}}^{\circ}$.)

Since each of these states $\Psi_{\gamma}$ depends only on a finite number of variables, borrowing the terminology from the quantum theory of free fields in Minkowski space, they are called cylindrical functions and denoted by Cyl. Gauge invariant cylindrical functions represent the 'typical' kinematic states. In many ways, Cyl is analogous to the space $C_{o}^{\infty}\left(R^{3}\right)$ of smooth functions of compact support on $R^{3}$ which is dense in the Hilbert space $L^{2}\left(R^{3}, d^{3} x\right)$ of quantum mechanics. Just as one often defines quantum operators - e.g., the position, the momentum and the Hamiltonians- on $C_{o}^{\infty}$ first and then extends them to an appropriately larger domain in the Hilbert space $L^{2}\left(R^{3}, d^{3} x\right)$, we will define our operators first on Cyl and then extend them appropriately.

Cylindrical functions provide considerable intuition about the nature of quantum states we are led to consider. These states represent 1-dimensional polymerlike excitations of geometry/gravity rather than 3-dimensional wavy undulations on flat space. Just as a polymer, although intrinsically 1-dimensional, exhibits 3dimensional properties in sufficiently complex and densely packed configurations, 
the fundamental 1-dimensional excitations of geometry can be packed appropriately to provide a geometry which, when coarse-grained on scales much larger than the Planck length, lead us to continuum geometries [13, 14]. Thus, in this description, gravitons can arise only as approximate notions in the low energy regime [32]. At the basic level, states in $\tilde{\mathcal{H}}^{o}$ are fundamentally different from the Fock states of Minkowskian quantum field theories. The main reason is the underlying diffeomorphism invariance: In absence of a background geometry, it is not possible to introduce the familiar Gaussian measures and associated Fock spaces.

\subsection{Statement of the problem}

We can now outline the general strategy that will be followed in section 3 .

Recall that the classical configuration variable is an $S U(2)$ connection $A_{a}^{i}$ on a 3-manifold $\Sigma$, where $i$ is the $s u(2)$-internal index with respect to a basis $\tau_{i}$. Its conjugate momentum $E_{j}^{b}$ has the geometrical interpretation of an orthonormal triad with density weight one 25]. Therefore, geometrical observables -functionals of the 3 -metric- can be expressed in terms of this field $E_{i}^{a}$. Fix within the 3-manifold $\Sigma$ any analytic, finite 2-surface. The area $A_{S}$ of $S$ is a well-defined, real-valued function on the full phase space of general relativity (which happens to depend only on $E_{i}^{a}$ ). It is easy to verify that these kinematical observables can be expressed as:

$$
A_{S}:=\int_{S} d x^{1} \wedge d x^{2}\left[E_{i}^{3} E^{3 i}\right]^{\frac{1}{2}}
$$

where, for simplicity, we have used adapted coordinates such that $S$ is given by $x^{3}=0$, and $x^{1}, x^{2}$ parameterize $S$, and where the internal index $i$ is raised by a the inner product we use on $s u(2), k\left(\tau_{i}, \tau_{j}\right)=-2 \operatorname{Tr}\left(\tau_{i} \tau_{j}\right)$. Similarly, if $R$ is any 3dimensional open region within $\Sigma$, the associated volume is a function on the phase space given by:

$$
V_{R}:=\int_{R} d x^{1} \wedge d x^{2} \wedge d x^{3}\left[\frac{1}{3} \eta_{a b c} \epsilon^{i j k} E_{i}^{a} E_{j}^{b} E_{k}^{c}\right]^{\frac{1}{2}},
$$

where $\eta_{a b c}$ is the (metric independent, natural) Levi-Civita density of weight -1 on $\Sigma$. Our task is to find the corresponding operators on the kinematical Hilbert space $\tilde{\mathcal{H}}^{o}$ and investigate their properties.

There are several factors that make this task difficult. Intuitively, one would expect that $E_{i}^{a}(x)$ to be replaced by the 'operator-valued distribution' $-i \hbar G \delta / \delta A_{a}^{i}(x)$. (See the basic Poisson bracket relation (2.3). Unfortunately, the classical expression of $A_{S}$ involves square-roots of products of $E$ 's and hence the formal expression of the corresponding operator is badly divergent. One must introduce a suitable regularization scheme. However, we do not have at our disposal the usual machinery

\footnotetext{
${ }^{4}$ We assume that the underlying 3 -manifold $\Sigma$ is orientable. Hence, principal $S U(2)$ bundles over $\Sigma$ are all topologically trivial. Therefore, we can represent the $S U(2)$ connections on the bundle by a $s u(2)$-valued 1 -form on $\Sigma$. The matrices $\tau_{i}$ are anti-Hermitian, given, e.g., by $(-i / 2)$-times the Pauli matrices.
} 
of Minkowskian field theories and even the precise rules that are to underlie such a regularization are not apriori clear.

There are however certain basic expectations that we can use as guidelines: i) the resulting operators should be well-defined on a dense sub-space of $\tilde{\mathcal{H}}^{o}$; ii) their final expressions should be diffeomorphism covariant, and hence, in particular, independent of any background fields that may be used in the intermediate steps of the regularization procedure; and, iii) since the classical observables are real-valued, the operators should be self-adjoint. These expectations seem to be formidable at first. Indeed, these demands are rarely met even in Minkowskian field theories; in presence of interactions, it is extremely difficult to establish rigorously that physically interesting operators are well-defined and self-adjoint. As we will see, the reason why one can succeed in the present case is two-folds. First, the requirement of diffeomorphism covariance is a powerful restriction that severely limits the possibilities. Second, the background independent functional calculus is extremely well-suited for the problem and enables one to circumvent the various road blocks in subtle ways.

Our general strategy will be following. We will define the regulated versions of area and volume operators on the dense sub-space $\mathrm{Cyl}$ of cylindrical functions and show that they are essentially self-adjoint (i.e., admit unique self-adjoint extensions to $\tilde{\mathcal{H}}^{o}$ ). This task is further simplified because the operators leave each sub-space $\mathcal{H}_{\gamma}$ spanned by cylindrical functions associated with any one graph $\gamma$ invariant. This in effect reduces the field theory problem (i.e., one with an infinite number of degrees of freedom) to a quantum mechanics problem (in which there are only a finite number of degrees of freedom). Finally, the operators in fact leave invariant certain finite dimensional sub-space of $\mathcal{H}^{o}$ (associated with 'extended spin networks' [17]). This powerful simplification further reduces the task of investigating the properties of these operators; in effect, the quantum mechanical problem (in which the Hilbert space is still infinite dimensional) is further simplified to a problem involving spin systems (where the Hilbert space is finite dimensional). It is because of these simplifications that a detailed analysis becomes possible.

\section{Quantum Geometry}

Our task is to construct a well-defined operator $\hat{A}_{S}$ and $\hat{V}_{R}$ starting from the classical expressions (2.4, 2.5). As is usual in quantum field theory, we will begin with the formal expression obtained by replacing $E_{i}^{a}$ in $(2.4,2.5)$ by the corresponding operator valued distribution $\hat{E}_{i}^{a}$ and then regulate it to obtain the required operators. (For an early discussion of non-perturbative regularization, see, in particular, [33]). For brevity, we will discuss the area operators in some detail and then give the final result for the volume operators. Furthermore, to simplify the presentation, we will assume that $S$ is covered by a single chart of adapted coordinates. Extension to the general case is straightforward: one mimics the procedure used to define the

integral of a differential form over a manifold. That is, one takes advantage of the 
coordinates invariance of the the resulting 'local' operator and uses a partition of unity.

\section{1 regularization}

The first step in the regularization procedure is to smear (the operator analog of) $E_{i}^{3}(x)$ and point split the integrand in (2.4). Since in this integrand the point $x$ lies on the 2 -surface $S$, let us try to use a 2-dimensional smearing function. Let $f_{\epsilon}(x, y)$ be a 1-parameter family of fields on $S$ which tend to the $\delta(x, y)$ as $\epsilon$ tends to zero; i.e., such that

$$
\lim _{\epsilon \rightarrow 0} \int_{S} d^{2} y f_{\epsilon}\left(x^{1}, x^{2} ; y^{1}, y^{2}\right) g\left(y^{1}, y^{2}\right)=g\left(x^{1}, x^{2}\right),
$$

for all smooth densities $g$ of weight 1 and of compact support on $S$. (Thus, $f_{\epsilon}(x, y)$ is a density of weight 1 in $x$ and a function in $y$.) The smeared version of $E_{i}^{3}(x)$ will be defined to be:

$$
\left[E_{i}^{3}\right]_{f}(x):=\int_{S} d^{2} y f_{\epsilon}(x, y) E_{i}^{3}(y)
$$

so that, as $\epsilon$ tends to zero, $\left[E_{i}^{3}\right]_{f}$ tends to $E_{i}^{3}(x)$. The point-splitting strategy now provides a 'regularized expression' of area:

$$
\begin{aligned}
{\left[A_{S}\right]_{f} } & :=\int_{S} d^{2} x\left[\int_{S} d^{2} y, f_{\epsilon}(x, y) E_{i}^{3}(y) \int_{S} d^{2} z f_{\epsilon}(x, z) E^{3 i}(z)\right]^{\frac{1}{2}} \\
& =\int_{S} d^{2} x\left[\left[E_{i}^{3}\right]_{f}(x)\left[E^{3 i}\right]_{f}(x)\right]^{\frac{1}{2}}
\end{aligned}
$$

which will serve as the point of departure in the subsequent discussion. To simplify technicalities, we will assume that the smearing field $f_{\epsilon}(x, y)$ has the following additional properties for sufficiently small $\epsilon>0$ : i) for any given $y, f_{\epsilon}(x, y)$ has compact support in $x$ which shrinks uniformly to $y$; and, ii) $f_{\epsilon}(x, y)$ is non-negative. These conditions are very mild and we are thus left with a large class of regulators.f

First, let us fix a graph $\gamma$ and consider a cylindrical function $\Psi_{\gamma}$ on $\overline{\mathcal{A}}$,

$$
\Psi_{\gamma}(\bar{A})=\psi\left(\bar{A}\left(e_{1}\right), . ., \bar{A}\left(e_{N}\right)\right) \equiv \psi\left(g_{1}, \ldots, g_{n}\right)
$$

where, as before, $N$ is the total number of edges of $\gamma, g_{k}=\bar{A}\left(e_{k}\right)$ and where $\psi$ is a smooth function on $[S U(2)]^{N}$. One can show [17] that the action of the regulated triad operator on such a state is given by:

$$
\left[\hat{E}_{i}^{3}\right]_{f}(x) \cdot \Psi_{\gamma}=\frac{i \ell_{P}^{2}}{2}\left[\sum_{I=1}^{N} \kappa_{I} f_{\epsilon}\left(x, v_{\alpha_{I}}\right) X_{I}^{i}\right] \cdot \psi\left(g_{1}, \ldots, g_{N}\right)
$$

\footnotetext{
${ }^{5}$ For example, $f_{\epsilon}(x, y)$ can be constructed as follows. Take any non-negative function $f$ of compact support on $S$ such that $\int d^{2} x f(x)=1$ and set $f_{\epsilon}(x, y)=\left(1 / \epsilon^{2}\right) f((x-y) / \epsilon)$. Here, we have used the given chart to write $x-y$ and give $f_{\epsilon}(x, y)$ a density weight in $x$.
} 
Here, $X_{I}^{i}$ are the left/right invariant vector fields on the $I$ th group copy in the argument of $\psi$ in the $i$ th internal direction, i.e., are operators assigned to the edge $e_{I}$ by the following formula

$$
X_{I}^{i} \cdot \psi\left(g_{1}, \ldots, g_{N}\right)= \begin{cases}\left(g_{I} \tau^{i}\right)_{B}^{A} \frac{\partial \psi}{\partial\left(g_{I}\right)_{B}^{A}}, & \text { when } e_{I} \text { is outgoing } \\ -\left(\tau^{i} g_{I}\right)_{B}^{A} \frac{\partial \psi}{\partial\left(g_{I}\right)_{B}^{A}}, & \text { when } e_{I} \text { is incoming }\end{cases}
$$

and $\kappa_{I}$ are real numbers given by:

$$
\kappa_{I}= \begin{cases}0, & \text { if } e_{I} \text { is tangential to } S \text { or does not intersect } S \\ +1, & \text { if } e_{I} \text { has an isolated intersection with } S \text { and lies above } S \\ -1, & \text { if } e_{I} \text { has an isolated intersection with } S \text { and lies below } S\end{cases}
$$

The right side again defines a cylindrical function based on the (same) graph $\gamma$. Denote by $\mathcal{H}_{\gamma}^{o}$ the Hilbert space $L^{2}\left(\mathcal{A}_{\gamma}, d \mu_{\gamma}^{o}\right)$ of square integrable cylindrical functions associated with a fixed graph $\gamma$. Since $\mu_{\gamma}^{o}$ is the induced Haar measure on $\mathcal{A}_{\gamma}$ and since the operator is just a sum of right/left invariant vector fields, standard results in analysis imply that, with domain $\mathrm{Cyl}_{\gamma}^{1}$ of all $C^{1}$ cylindrical functions based on $\gamma$, it is an essentially self-adjoint on $\mathcal{H}_{\gamma}^{o}$. Now, it is straightforward to verify that the operators on $\mathcal{H}_{\gamma}^{o}$ obtained by varying $\gamma$ are all compatible ${ }^{0}$ in the appropriate sense. Hence, it follows from the general results in $[9]$ that $\left[\hat{E}_{i}^{3}\right]_{f}(x)$, with domain $\mathrm{Cyl}^{1}$ (the space of all $C^{1}$ cylindrical functions), is an essentially self-adjoint operator on $\mathcal{H}^{o}$. For notational simplicity, we will denote its self-adjoint extension also by $\left[\hat{E}_{i}^{3}\right]_{f}(x)$. (The context should make it clear whether we are referring to the essentially self-adjoint operator or its extension.)

Let us now turn to the integrand of the smeared area operator (corresponding to $(3.3))$. Denoting the determinant of the intrinsic metric on $S$ by $g_{S}$, we have:

$$
\begin{aligned}
{\left[\hat{g}_{S}\right]_{f}(x) \cdot \Psi_{\gamma} } & :=\left[E_{i}^{3}\right]_{f}(x)\left[E^{3 i}\right]_{f}(x) \cdot \Psi_{\gamma} \\
& =-\frac{\ell_{P}^{4}}{4}\left[\sum_{I, J} \kappa(I, J) f_{\epsilon}\left(x, v_{\alpha_{I}}\right) f_{\epsilon}\left(x, v_{\alpha_{J}}\right) X_{I}^{i} X_{J}^{i}\right] \cdot \Psi_{\gamma}
\end{aligned}
$$

where the summation goes over all the oriented pairs $(I, J) ; v_{\alpha_{I}}$ and $v_{\alpha_{J}}$ are the vertices at which edges $e_{I}$ and $e_{J}$ intersect $S ; \kappa(I, J)=\kappa_{I} \kappa_{J}$ equals 0 if either of the two edges $e_{I}$ and $e_{J}$ fails to intersect $S$ or lies entirely in $S,+1$ if they lie on the same side of $S$, and, -1 if they lie on the opposite sides. (For notational simplicity, from now on we shall not keep track of the position of the internal indices $i$; as noted in Sec. 2.3, they are contracted using the invariant metric on the Lie algebra $s u(2)$.) The next step is to consider vertices $v_{\alpha}$ at which $\gamma$ intersects $S$ and simply

\footnotetext{
${ }^{6}$ Given two graphs, $\gamma$ and $\gamma^{\prime}$, we say that $\gamma \geq \gamma^{\prime}$ if and only if every edge of $\gamma^{\prime}$ can be written as a composition of edges of $\gamma$. Given two such graphs, there is a projection map from $\mathcal{A}_{\gamma}$ to $\mathcal{A}_{\gamma^{\prime}}$, which, via pull-back, provides an unitary embedding $U_{\gamma, \gamma^{\prime}}$ of $\tilde{\mathcal{H}}_{\gamma^{\prime}}^{o}$ into $\tilde{\mathcal{H}}_{\gamma}^{o}$. A family of operators $\mathcal{O}_{\gamma}$ on the Hilbert spaces $H o_{\gamma}$ is said to be compatible if $U_{\gamma, \gamma^{\prime}} \mathcal{O}_{\gamma^{\prime}}=\mathcal{O}_{\gamma} U_{\gamma, \gamma^{\prime}}$ and $U_{\gamma, \gamma^{\prime}} D_{\gamma^{\prime}} \subset D_{\gamma}$ for all $g \geq g^{\prime}$.
} 
rewrite the above sum by re-grouping terms by vertices. The result simplifies if we choose $\epsilon$ sufficiently small so that, $f_{\epsilon}\left(x, v_{\alpha_{I}}\right) f_{\epsilon}\left(x, v_{\alpha_{J}}\right)$ is zero unless $v_{\alpha_{I}}=v_{\alpha_{J}}$. We then have:

$$
\left[\hat{g}_{S}\right]_{f}(x) \cdot \Psi_{\gamma}=-\frac{\ell_{P}^{4}}{4}\left[\sum_{\alpha}\left(f_{\epsilon}\left(x, v_{\alpha}\right)\right)^{2} \sum_{I_{\alpha}, J_{\alpha}} \kappa\left(I_{\alpha}, J_{\alpha}\right) X_{I_{\alpha}}^{i} X_{J_{\alpha}}^{i}\right] \cdot \Psi_{\gamma},
$$

where the index $\alpha$ labels the vertices on $S$ and $I_{\alpha}$ and $J_{\alpha}$ label the edges at the vertex $\alpha$.

The next step is to take the square-root of this expression. The same reasoning that established the self-adjointness of $\left[\hat{E}_{i}^{3}\right]_{f}(x)$ now implies that $\left[\hat{g}_{S}\right]_{f}(x)$ is a nonnegative self-adjoint operator and hence has a well-defined square-root which is also a positive definite self-adjoint operator. Since we have chosen $\epsilon$ to be sufficiently small, for any given point $x$ in $S, f_{\epsilon}\left(x, v_{\alpha}\right)$ is non-zero for at most one vertex $v_{\alpha}$. We can therefore take the sum over $\alpha$ outside the square-root. One then obtains

$$
\left(\left[\hat{g}_{S}\right]_{f}\right)^{\frac{1}{2}}(x) \cdot \Psi_{\gamma}=\frac{\ell_{P}^{2}}{2} \sum_{\alpha} f_{\epsilon}\left(x, v_{\alpha}\right)\left[\sum_{I_{\alpha}, J_{\alpha}} \kappa\left(I_{\alpha}, J_{\alpha}\right) X_{I_{\alpha}}^{i} X_{J_{\alpha}}^{i}\right]^{\frac{1}{2}} \cdot \Psi_{\gamma} .
$$

Note that the operator is neatly split; the $x$-dependence all resides in $f_{\epsilon}$ and the operator within the square-root is 'internal' in the sense that it acts only on copies of $S U(2)$.

Finally, we can remove the regulator, i.e., take the limit as $\epsilon$ tends to zero. By integrating both sides against test functions on $S$ and then taking the limit, we conclude that the following equality holds in the distributional sense:

$$
\widehat{\sqrt{g_{S}}}(x) \cdot \Psi_{\gamma}=\frac{\ell_{P}^{2}}{2} \sum_{\alpha} \delta^{(2)}\left(x, v_{\alpha}\right)\left[\sum_{I_{\alpha}, J_{\alpha}} \kappa\left(I_{\alpha}, J_{\alpha}\right) X_{I_{\alpha}}^{i} X_{J_{\alpha}}^{i}\right]^{\frac{1}{2}} \cdot \Psi_{\gamma} .
$$

Hence, the regularized area operator is given by:

$$
\hat{A}_{S} \cdot \Psi_{\gamma}=\frac{\ell_{P}^{2}}{2} \sum_{\alpha}\left[\sum_{I_{\alpha}, J_{\alpha}} \kappa\left(I_{\alpha}, J_{\alpha}\right) X_{I_{\alpha}}^{i} X_{J_{\alpha}}^{i}\right]^{\frac{1}{2}} \cdot \Psi_{\gamma}
$$

(Here, as before, $\alpha$ labels the vertices at which $\gamma$ intersects $S$ and $I_{\alpha}$ labels the edges of $\gamma$ at the vertex $v_{\alpha}$.) With $\mathrm{Cyl}^{2}$ as its domain, $\hat{A}_{S}$ is essentially self-adjoint on the Hilbert space $\mathcal{H}^{o}$.

The classical expression $A_{S}$ of (2.4) is a rather complicated. It is therefore somewhat surprising that the corresponding quantum operators can be constructed rigorously and have quite manageable expressions. The essential reason is the underlying diffeomorphism invariance which severely restricts the possible operators. Given a surface and a graph, the only diffeomorphism invariant entities are the intersection vertices. Thus, a diffeomorphism covariant operator can only involve structure at these vertices. In our case, it just acts on the copies of $S U(2)$ associated with various edges at these vertices. 
We will close this discussion by simply writing the final expression of the volume operator:

$$
\hat{V}_{R} \cdot \Psi_{\gamma}:=\frac{\ell_{P}^{3}}{4 \sqrt{3}} \sum_{\alpha}\left|\sum_{I_{\alpha}, J_{\alpha}, K_{\alpha}} i \epsilon^{i j k} \epsilon\left(I_{\alpha}, J_{\alpha}, K_{\alpha}\right) X_{I_{\alpha}}^{i} X_{J_{\alpha}}^{j} X_{K_{\alpha}}^{k}\right|^{\frac{1}{2}} \cdot \Psi_{\gamma},
$$

where the first sum now is over vertices which lie in the region $R$ and $\epsilon\left(I_{\alpha}, J_{\alpha}, K_{\alpha}\right)$ is 0 if the three edges are linearly dependent at the vertex $v_{\alpha}$ and otherwise \pm 1 depending on the orientation they define. With $\mathrm{Cyl}^{3}$ as its domain, $\hat{V}_{R}$ is essentially self-adjoint on $\mathcal{H}^{o}$.

To summarize, the diffeomorphism covariant functional calculus has enabled us to regulate the area and volume operators. While in the intermediate steps we have used additional structures - such as charts- the final results make no reference to these structures; the final expressions of the quantum operators have the same covariance properties as those of their classical counterparts.

\subsection{General properties of Geometric operators}

We will now discuss the key properties of these geometric operators and point out a few subtleties. As in the previous subsection, for definiteness, the detailed comments will refer to the area operators. It should be clear from the discussion that analogous remarks hold for the volume operators as well.

1. Discreteness of the spectrum: By inspection, it follows that the total area operator $\hat{A}_{S}$ leaves the sub-space of $\mathrm{Cyl}_{\gamma}^{2}$ which is associated with any one graph $\gamma$ invariant and is a self-adjoint operator on the sub-space $\mathcal{H}_{\gamma}^{o}$ of $\mathcal{H}^{o}$ corresponding to $\gamma$. Next, recall that $\mathcal{H}_{\gamma}^{o}=L^{2}\left(\mathcal{A}_{\gamma}, d \mu^{o}\right)$, where $\mathcal{A}_{\gamma}$ is a compact manifold, isomorphic with $(S U(2))^{N}$ where $N$ is the total number of edges in $\gamma$. The restriction of $\hat{A}_{S}$ to $\mathcal{H}_{\gamma}^{o}$ is given by certain commuting elliptic differential operators on this compact manifold. Therefore, all its eigenvalues are discrete. Now suppose that the complete spectrum of $\hat{A}_{S}$ on $\mathcal{H}^{o}$ has a continuous part. Denote by $P_{c}$ the associated projector. Then, given any $\Psi$ in $\mathcal{H}^{o}, P_{c} \cdot \Psi$ is orthogonal to $\mathcal{H}_{\gamma}^{o}$ for any graph $\gamma$, and hence to the space Cyl of cylindrical functions. Since $\mathrm{Cyl}^{2}$ is dense in $\mathcal{H}^{o}, P_{c} \cdot \Psi$ must vanish for all $\Psi$ in $\mathcal{H}^{o}$. Hence, the spectrum of $\hat{A}_{S}$ has no continuous part.

Note that this method is rather general: It can be used to show that any selfadjoint operator on $\mathcal{H}^{o}$ which maps (the intersection of its domain with) $\mathcal{H}_{\gamma}^{o}$ to $\mathcal{H}_{\gamma}^{o}$, and whose action on $\mathcal{H}_{\gamma}^{o}$ is given by elliptic differential operators, has a purely discrete spectrum on $\mathcal{H}^{o}$. Geometrical operators, constructed purely from the triad field tend to satisfy these properties.

In the case of area operators, one can do more: complete spectrum has been calculated. The eigenvalues are given by [17]:

$$
a_{S}=\frac{\ell_{P}^{2}}{2} \sum_{\alpha}\left[2 j_{\alpha}^{(d)}\left(j_{\alpha}^{(d)}+1\right)+2 j_{\alpha}^{(u)}\left(j_{\alpha}^{(u)}+1\right)-j_{\alpha}^{(d+u)}\left(j_{\alpha}^{(d+u)}+1\right)\right]^{\frac{1}{2}}
$$


where $\alpha$ labels a finite set of points in $S$ and the non-negative half-integers assigned to each $\alpha$ are subject to the inequality

$$
j^{(d)}+j^{(u)} \geq j^{(d+u)} \geq\left|j^{(d)}-j^{(u)}\right| .
$$

There is, in particular, the smallest, non-zero eigenvalue, the 'elementary quantum of area': $a_{S}^{o}=(\sqrt{3} / 4) \ell_{P}^{2}$. Note, however, that the level spacing between eigenvalues is not regular. For large $a_{S}$, the difference between consecutive eigenvalues in fact goes to zero as $1 / \sqrt{a_{S}}$. (For comparison with other results [13, 20], see [17].)

2. Area element: Note that not only is the total area operator well-defined, but in fact it arises from a local area element, $\widehat{\sqrt{g}}$, which is an operator-valued distribution in the usual sense. Thus, if we integrate it against test functions, the operator is densely defined on $\mathcal{H}^{o}$ (with $C^{2}$ cylindrical functions as domain) and the matrix elements

$$
\left\langle\Psi_{\gamma^{\prime}}^{\prime}, \widehat{\sqrt{g_{S}}}(x) \cdot \Psi_{\gamma}\right\rangle
$$

are 2-dimensional distributions on $S$. Furthermore, since we did not have to renormalize the regularized operator (3.10) before removing the regulator, there are no free renormalization constants involved. The local operator is completely unambiguous.

3. $\left[\hat{g}_{S}\right]_{f}$ versus its square-root: Although the regulated operator $\left[\hat{g}_{s}\right]_{f}$ is welldefined, if we let $\epsilon$ to go zero, the resulting operator is in fact divergent: roughly, it would lead to the square of the 2-dimensional $\delta$ distribution. Thus, the determinant of the 2-metric is not a well-defined in the quantum theory. As we saw, however, the square-root of the determinant is well defined: We have to first take the square-root of the regulated expression and then remove the regulator. This, in effect, is the essence of the regularization procedure.

To get around this divergence of $\hat{g}_{S}$, as is common in Minkowskian field theories, we could have first rescaled $\left.\left[\hat{g}_{S}\right]_{f}\right]$ by an appropriate factor and then taken the limit. Then result can be a well-defined operator, but it will depend on the choice of the regulator, i.e., additional structure introduced in the procedure. Indeed, if the resulting operator is to have the same density character as its classical analog $g_{S}(x)$-which is a scalar density of weight two- then the operator can not respect the underlying diffeomorphism invariance. For, there is no metric/chart independent distribution on $S$ of density weight two. Hence, such a 'renormalized' operator is not useful to a fully non-perturbative approach. For the square-root, on the other hand, we need a local density of weight one. And, the 2-dimensional Dirac distribution provides this; now is no apriori obstruction for a satisfactory operator corresponding to the area element to exist. This is an illustration of what appears to be typical in non-perturbative approaches to quantum gravity: Either the limit of the operator exists as the regulator is removed without the need of renormalization or it inherits back-ground dependent renormalization fields (rather than constants).

4. Gauge invariance: The classical area element $\sqrt{g_{S}}$ is invariant under the internal rotations of triads $E_{i}^{a}$; its Poisson bracket with the Gauss constraint functional 
vanishes. This symmetry is preserved in the quantum theory: the quantum operator $\widehat{\sqrt{g_{S}}}$ commutes with the induced action of $\overline{\mathcal{G}}$ on the Hilbert space $\mathcal{H}^{\circ}$. Thus, $\sqrt{g_{S}}$ and the total area operator $\hat{A}_{S}$ map the space of gauge invariant states to itself; they project down to the Hilbert space $\tilde{\mathcal{H}}^{o}$ of kinematic states. In the classical theory, the allowed values of the area operators on the full phase space are the same as those on the constraint surface. That is, the passage from all kinematical states to the dynamically permissible ones does not give rise to restrictions on the "classical spectrum' of these operators. The same is true in the quantum theory. The spectrum of $\hat{A}_{S}$ on $\tilde{\mathcal{H}}^{o}$ is the same as that on $\mathcal{H}^{o}$. (Only the degeneracies of eigenvectors changes.)

\section{Discussion}

In section 1, we began by formulating what we mean by quantization of geometry: Are there geometrical observables which assume continuous values on the classical phase space but whose quantum analogs have discrete spectra? In order to explore these issues, we had to use a fully non-perturbative framework which does not use a background geometry. In the last two sections, we answered the question in the affirmative in the case of area and volume operators. The discreteness came about because, at the microscopic level, geometry has a distributional character with 1-dimensional excitations. This is the case even in semi-classical states which approximate classical geometries macroscopically [13, [14]. We wish to emphasize that these results have been obtained in the framework of a (non-traditional but) rigorous quantum field theory. In particular, the issues of regularization have been addressed squarely and the calculations are free of hidden infinities.

We will conclude by examining the main results from various angles.

1. Inputs: The picture of quantum geometry that has emerged here is strikingly different from the one in perturbative, Fock quantization. Let us begin by recalling the essential ingredients that led us to the new picture.

This task is made simpler by the fact that the new functional calculus provides the degree of control necessary to distill the key assumptions. There are only two essential inputs. The first assumption is that the Wilson loop variables, $T_{\alpha}=$ $\operatorname{Tr} \mathcal{P} \exp \int_{-} \alpha A$, should serve as the configuration variables of the theory, i.e., that the Hilbert space of (kinematic) quantum states should carry a representation of the $C^{\star}$-algebra generated by the Wilson loop functionals on the classical configuration space $\mathcal{A} / \mathcal{G}$. The second assumption singles out the measure $\tilde{\mu}^{o}$. In essence, if we assume that $\hat{E}_{i}^{a}$ be represented by $-i \hbar \delta / \delta A_{a}^{i}$, the 'reality conditions' lead us to the measure $\tilde{\mu}^{o}$ [11]. Both these assumptions seem natural from a mathematical physics perspective. However, a deeper understanding of their physical meaning is still needed for a better understanding of the overall situation.']

\footnotetext{
${ }^{7}$ In particular, in the standard spin-2 Fock representation, one uses quite a different algebra of configuration variables and uses the flat background metric to represent it. It then turns out that
} 
2. Kinematics versus Dynamics: As was emphasized in the main text, in the classical theory, geometrical observables are defined as functionals on the full phase space; these are kinematical quantities whose definitions are quite insensitive to the precise nature of dynamics, presence of matter fields, etc. Thus, in the connection dynamics description, all one needs is the presence of a canonically conjugate pair consisting of a connection and a (density weighted) triad. Therefore, one would expect the result on the area operator presented here to be quite robust. In particular, they should continue to hold if we bring in matter fields or extend the theory to supergravity.

There is, however, a subtle caveat: In field theory, one can not completely separate kinematics and dynamics. For instance, in Minkowskian field theories, the kinematic field algebra typically admits an infinite number of inequivalent representations and a given Hamiltonian may not be meaningful on a given representation. Therefore, whether the kinematical results obtained in any one representation actually hold in the physical theory depends on whether that representation supports the Hamiltonian of the model. In the present case, therefore, a key question is whether the quantum constraints of the theory can be imposed meaningfully on $\tilde{\mathcal{H}}^{o}$. 8 Results to date indicate (but do not yet conclusively prove) that this is likely to be the case for general relativity. The general expectation is that this would be the case also for a class of theories such as supergravity, which are 'near' general relativity. The results obtained here would continue to be applicable for this class of theories.

3. Dirac Observable: Note that $\hat{A}_{S}$ has been defined for any surface $S$. Therefore, these operators will not commute with constraints; they are not Dirac observables. To obtain a Dirac observable, one would have to specify $S$ intrinsically, using, for example, matter fields. In view of the Hamiltonian constraint, the problem of providing an explicit specification is extremely difficult. However, this is true already in the classical theory. In spite of this, in practice we do manage to specify surfaces (or 3-dimensional regions) and furthermore compute their areas (volumes) using the standard formula from Riemannian geometry which is quite insensitive to the details of how the surface (region) was actually defined. Similarly, in the quantum theory, if we could specify a surface $S$ (region $R$ ) intrinsically, we could compute the spectrum of $\hat{A}_{S}$ and $\hat{V}_{R}$ using results obtained in this paper.

4. Manifold versus Geometry: In this paper, we began with an orientable, analytic, 3-manifold $\Sigma$ and this structure survives in the final description. As noted in footnote 1 , we believe that the assumption of analyticity can be weakened without changing the qualitative results. Nonetheless, a smoothness structure of the underlying manifold will persist. What is quantized is 'geometry' and not smoothness.

the Wilson loops are not represented by well-defined operators; our first assumption is violated. One can argue that in a fully non-perturbative context, one can not mimic the Fock space strategy. Further work is needed, however, to make this argument water-tight.

${ }^{8}$ Note that this issue arises in any representation once a sufficient degree of precision is reached. In geometrodynamics, this issue is not discussed simply because generally the discussion is rather formal. 
Now, in $2+1$ dimensions, using the loop representation one can recast the final description in a purely combinatorial fashion (at least in the so-called 'time-like sector' of the theory). In this description, at a fundamental level, one can avoid all references to the underlying manifold and work with certain abstract groups which, later on, turn out to be the homotopy groups of the 'reconstructed/derived' 2-manifold (see, e.g., section 3 in [30]). One might imagine that if and when our understanding of knot theory becomes sufficiently mature, one would also be able to get rid of the underlying manifold in the $3+1$ theory and introduce it later as a secondary/derived concept. At present, however, we are quite far from achieving this.

In the context of geometry, however, a detailed combinatorial picture is emerging. Geometrical quantities are being computed by counting; integrals for areas and volumes are being reduced to genuine sums. (However, the sums are not the 'obvious' ones, often used in approaches that begin by postulating underlying discrete structures. In the computation of area, for example, one does not just count the number of intersections; there are precise and rather intricate algebraic factors that depend on the representations of $S U(2)$ associated with the edges at each intersection.) It is striking to note that, in the same address [4] in which Riemann first raised the possibility that geometry of space may be a physical entity, he also introduced ideas on discrete geometry. The current program comes surprisingly close to providing us with a concrete realization of these ideas.

To summarize, it is possible to do physics in absence of a background space-time geometry. It does require the use of new mathematical methods, such as a diffeomorphism covariant functional calculus. However, one can obtain concrete, physically motivated results which are quite surprising from the viewpoint of Minkowskian field theories.

\section{Acknowledgments}

We would also like to thank John Baez, Bernd Bruegman, Don Marolf, Jose Mourao, Thomas Thiemann, Lee Smolin, John Stachel and especially Carlo Rovelli for discussions. This work was supported in part by the NSF Grants PHY93-96246 and PHY95-14240, the KBN grant 2-P302 11207 and by the Eberly fund of the Pennsylvania State University. JL thanks the members of the Max Planck Institute for their hospitality.

\section{References}

[1] A. S. Wightman, The usefulness of a general theory of quantized fields.

[2] B. S. DeWitt, Quantum field theory and spacetime-formalism and reality.

[3] C. Rovelli, How much of quantum field theory is compatible with what we know about spacetime? 
[4] B. Riemann, Über die Hypothesen, welche der Geometrie zugrunde liegen (1854).

[5] A. Ashtekar and C.J. Isham, Class. \& Quan. Grav. 9, 1433 (1992).

[6] A. Ashtekar and J. Lewandowski, "Representation theory of analytic holonomy $C^{\star}$ algebras", in Knots and quantum gravity, J. Baez (ed), (Oxford University Press, Oxford 1994); J. Math. Phys. 36, 2170 (1995).

[7] J. Baez, Lett. Math. Phys. 31, 213 (1994); "Diffeomorphism invariant generalized measures on the space of connections modulo gauge transformations", hep-th/9305045, in the Proceedings of the conference on quantum topology, D. Yetter (ed) (World Scientific, Singapore, 1994).

[8] D. Marolf and J. M. Mourão, Commun. Math. Phys. 170, 583 (1995).

[9] A. Ashtekar and J. Lewandowski, J. Geo. \& Phys. 17, 191 (1995).

[10] J. Baez, "Spin network states in gauge theory", Adv. Math. (in press); "Spin networks in non-perturbative quantum gravity," pre-print gr-qc/9504036.

[11] A. Ashtekar, J. Lewandowski, D. Marolf, J. Mourão and T. Thiemann, J. Math. Phys. 36, 6456 (1995).

[12] A. Ashtekar, J. Lewandowski, D. Marolf, J. Mourão and T. Thiemann, J. Funct. Analysis, 135, 519 (1996).

[13] A. Ashtekar, C. Rovelli and L. Smolin, Phys. Rev. Lett. 69, 237 (1992).

[14] A. Ashtekar and L. Bombelli (in preparation)

[15] J. Beckenstein and V.F. Mukhanov, Spectroscopy of quantum black holes, preprint gr-qc/9505012.

[16] S. Carlip, Class. \& Quantuntum Grav. 12, 2853 (1995); Statistical mechanics and black-hole entropy, preprint gr-qc/9509024.

[17] A. Ashtekar and J. Lewandowski, Quantum theory of geometry I: Area operators, gr-qc 9602046; Class. \& Quan. Grav. (to appear)

[18] A. Ashtekar and J. Lewandowski, Quantum thory of geometry II: 3-dimensional operators (in preparation).

[19] J. Lewandowski, Volume and quantizations, pre-print, gr-qc/9602035

[20] C. Rovelli and L. Smolin, Nucl. Phys. B442, 593 (1995).

[21] R. Loll, Phys. Rev. Lett. 75, 3084 (1995). 
[22] R. Loll, Spectrum of the volume operator in quantum gravity, Nucl. Phys. B (to appear).

[23] C. Rovelli, L. Smolin, Nucl. Phys. B331, 80 (1990).

[24] A. Ashtekar, Polymer geometry at Planck scale and quantum Einstein's equations, Acta Cosmologica XXI, 85-110 (1996); CGPG-95/11-5, hep-th/9601054.

[25] A. Ashtekar, Phys. Rev. Lett. 57, 2244 (1986); Phys. Rev. D36, 1587 (1987);

[26] A. Ashtekar, in Mathematics and General Relativity, ed J. Isenberg (American Mathematical Society, Providence, 1987).

[27] A. Ashtekar, Lectures on Non-perturbative Canonical Gravity, Notes prepared in collaboration with R.S. Tate (World Scientific, Singapore, 1991).

[28] T. Thiemann, Reality conditions inducing transforms for quantum gauge field theory and quantum gravity, CGPG-95/11-4 pre-print.

[29] A. Ashtekar, A Generalized Wick transform for gravity CGPG-95/12-1 preprint.

[30] A. Ashtekar, in Gravitation and Quantizations, ed B. Julia and J. Zinn-Justin (Elsevier, Amsterdam, 1995).

[31] J. Baez and S. Sawin, Functional integration on spaces of connections, $\mathrm{q}$ $\operatorname{alg} / 9507023 ;$ J. Lewandowski and T. Thiemann (in preparation)

[32] J. Iwasaki and C. Rovelli, Int. J. Modern. Phys. D1, 533 (1993); Class. Quant. Grav. 11, 2899 (1994).

[33] B. Bruegman and J. Pullin, Nucl.Phys., B390, 399 (1993). 